\title{
Trabajadores pobres en España. El contexto de la crisis económica como marco para comprender la desigualdad
}

\author{
Working poor in Spain. The context of the economic crisis \\ as a framework for understanding inequality
}

\author{
Rafael Martínez-Martín, José Manuel García-Moreno, \\ Antonio Manuel Lozano-Martín
}

Departamento de Sociología de la Universidad de Granada, España

\section{Resumen}

En el presente artículo estudiaremos las condiciones de vida y la posición que ocupa en la estructura social el trabajador pobre en España. Tras contextualizar el concepto de trabajador pobre en el ámbito de la precariedad laboral y de la configuración actual de los mercados de trabajo en términos duales - segmentados, el análisis de los datos de la Encuesta de Condiciones de Vida y de la Encuesta Anual de Estructura Salarial (ambas del Instituto Nacional de Estadística - INE) nos permite concluir que, para el caso español entre 2008 y hasta 2014 - 2015, se han producido importantes variaciones tanto en la renta media anual por diversas variables, como en las ganancias medias por persona, así como un incremento en los indicadores de riesgo de pobreza, y de las personas viviendo en hogares de baja intensidad laboral.

Palabras clave: Trabajador pobre, precariedad, mercados de trabajo, riesgo de pobreza.

Abstract

In the presente article we study working poor living conditions and social position in social estructure in Spain. After contextualize the concept of working por in the frame or labour precariousness and the current configuration of labor markets in dual - segmented terms, the data analysis from the Survey of Living Conditions and the Annual Survey of Salary Structure (both from the National Institute of Statistics - INE) allows us to conclude that, for Spanish case, there have been significant variations in both the average annual income per differente variables, and the average earnings per person and, therefore an increase in poverty risk indicators as well as an increase in the number of people living in low labor-intensive households.

Keywords: Working poor, insecurity, labor markets, poverty risk. 


\section{INTRODUCCIÓN}

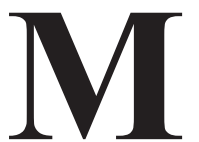

ás allá de los sistemas de medición propiamente economicistas, desde la perspectiva sociológica hemos desarrollado una forma de entender el mundo del trabajo que podemos considerar propia. Señalamos así en la dirección de una triple perspectiva analítica desde la que enfocamos este artículo y que es tremendamente útil a efectos de nuestro objeto de estudio: los trabajadores pobres. En primer lugar, es de nuestro interés conocer qué papel cumple el trabajo desde el punto de vista de la biografía de los individuos, junto con la educación, las situaciones desempleo y la conocida como compatibilidad de la vida familiar y la vida laboral. En segundo lugar, manejamos una perspectiva analítica del trabajo en el que se ahonda en las desigualdades que nacen en el propio mercado de trabajo, desigualdades que se construyen por razón de género, de edad, por regiones, por estrato urbano o rural, por nacionalidad, por el nivel socioeconómico individual o como consecuencia de las propias situaciones familiares, de las propias situaciones de los hogares. Finalmente, una tercera perspectiva, desde la cual podemos profundizar en las diferencias que el propio mercado de trabajo provoca en la estructura social y, con ello, en la manera en la que los individuos pueden ser reconocidos en su distribución sobre la base de los indicadores de estratificación social (De Miguel, 1998).

Asimismo, es evidente que la sociedad actual sigue siendo una sociedad que se articula en torno al mundo laboral, y es una sociedad que se interpretaría en su complejidad a partir de la propia división social del trabajo. A este respecto, Giner (1979) señalaba con acierto que una sociedad con algún grado de complejidad sobrevive en la medida en la que los grupos que la constituyen, y dentro de ellos los individuos, se dividen en módulos que denomina módulos de especialización. Es decir, uniríamos división social del trabajo, especialización y centralidad del empleo como carácter definitorio de una sociedad, como es el caso de la española.

La mayor parte de nuestra vida se articula en torno al trabajo. La inserción social de los individuos no es comprensible sin la inserción laboral. El trabajo es germen del estatus social de los individuos y, con ello, de la posición que ocuparán en la estructura social. El trabajo respondería también a una necesidad básica de cualquier forma de estructuración social: permitiría a los individuos valorarse a sí mismos y ser valorados por el 
conjunto de la comunidad (Requena, 2001). Sin embargo, en situaciones próximas a la exclusión, como es el caso del trabajador pobre, el desajuste de expectativas entre lo que se quiere ser en sociedad y lo que se es en realidad, a partir de los indicadores de clasificación en dichas estructuras, dan cuenta de las dificultades y conflictos a los que se estaría enfrentando este tipo de trabajador en la actualidad.

Hablaremos así, en este artículo, del trabajo como un actor funcional desde el punto de vista social. Funcional en la medida en que cubre necesidades, unas más básicas y otras más propias de la realización personal y/o de integración social. Salanova, Hontangas y Peiró (1996) presentan de forma clara cuáles son los elementos positivos que genera el trabajo en los individuos. El trabajo cumple con una función económica, en la medida en la que permite (en el caso de los trabajadores pobres, esta función estaría en riesgo) obtener un salario para la mantener niveles de consumo óptimos a la sociedad actual alejados de los riesgos de pobreza y exclusión social. Junto con esta función, señalan otras que también son esenciales y que, por supuesto, también están en conflicto con el perfil de trabajador objeto de esta investigación. Así, el trabajo proporciona estatus y prestigio social y, como consecuencia de ello, el trabajo cumple con una función integrativa o significativa frente al desempleado o subempleado que perdería las referencias sociales básicas relacionadas con su posición en la estructura. Además, el trabajo es fuente de identidad personal, puesto que lo que somos y cómo nos ven los demás, tal y como ya hemos indicado, se materializa en una parte importante, en el entorno laboral. Ese entorno laboral es el contexto en el cual se generan oportunidades para la interacción y los contactos sociales, manteniendo a los individuos en una actividad más o menos obligatoria y permitiendo que, en él, dichos individuos desarrollen habilidades y destrezas. El trabajo, además, transmite normas, creencias $y$ expectativas sociales $\mathrm{y}$, junto a todas estas funciones, el trabajo permite estructurar el tiempo.

Por tanto, el valor social del trabajo parece claro y, además, está en la base de la producción de una cultura específica, una cultura que denominaríamos cultura productiva (Bericat, 1989), una cultura en la que el trabajo actúa de forma determinante en la creación de espacios vitales y en la producción de experiencias que tienen significado para aquellos que viven insertos laboralmente (Valenzuela, Reygadas y Cruces, 2015) y que, por el contrario, estarían en peligro para aquellos trabajadores que están en situación de pobreza laboral en mercados de trabajo cada vez más precarios. 


\section{LA PRECARIEDAD LABORAL COMO CONTEXTO}

Sin lugar a dudas, el escenario de intercambio de oferta y demanda de trabajo que se describe en el contexto de cualquier mercado laboral es un escenario imperfecto. Hablar de la imperfección de estos mercados, es señalar las interferencias de factores tanto externos como internos que lo mediatizan. Sarriés (1999) al hablar de las relaciones laborales, y del papel de los mercados de trabajo en la importancia dada o no al factor humano en los contextos organizativos empresariales, señala una serie de características de dichos mercados que nos ayudan a entender bien los procesos de precarización laboral que se han venido produciendo en las últimas décadas.

Así, los mercados de trabajo son mercados regulados, en la medida en la que no sólo se regula un derecho laboral sino cualquier proceso de flexibilización del mismo. Son mercados intervenidos por cuanto son los gobiernos, con el margen de maniobra de que disponen, los que pueden actuar para generar empleo o para favorecer la contratación o no de determinados perfiles laborales. Pero es la flexibilidad aludida ya, la que en mayor medida caracteriza los mercados laborales actuales. Flexibilidad en la línea de la modificación de los tipos de contratación, abaratamiento del despido, despenalización del despido, reducción de cargas empresariales a la seguridad social, etc. Es así, y en este marco general, en el que los mercados laborales actuales son discriminatorios (segmentos de población discriminados por el simple hecho de pertenecer a ese segmento: jóvenes, mujeres, parados de larga duración, etc.) y opacos en la medida en la que la economía sumergida genera todo un entramado laboral informal más allá del control legal. Es en esos escenarios más precarios en los que toma cuerpo la realidad del trabajador pobre.

Partiendo de esta realidad, llegamos a otra, la de la precariedad laboral. En este sentido, y al hablar de las transformaciones producidas en las sociedades occidentales postindustriales, sobre la base de la descualificación del trabajador industrial, y con el consiguiente riesgo de exclusión de dichos trabajadores, Dahrendorf expresaba de forma clara que,

por un lado, el progreso técnico requiere una cualificación superior (...) y, por otro lado, suprime empleos, dejando fuera de juego a los que no han podido reciclarse por falta de oportunidades o talento (...) Los trabajadores cualificados de las industrias mecánicas pasan a ser trabajadores no cualificados en la industria electrónica, en obreros que pronto pasarán a ser eventuales, después simples parados y, finalmente, quedarán condenados al paro perpetuo (Dahrendorf, 2003: 128). 
En este sentido, lo que estamos señalando es la distinción, cada vez más profunda, cada vez más afianzada en mercados laborales como es el caso del español, entre empleo estándar y empleo atípico (Rodgers y Rodgers, 1992). El empleo estándar es aquel que se materializa sobre la negociación colectiva y que desemboca en protección y estabilidad para el trabajador, regularidad y seguridad en el puesto de trabajo. Por el contrario, el empleo atípico es aquel que se aleja del estándar y, como consecuencia de ese distanciamiento, quedará caracterizado por la temporalidad en los contratos, la eventualidad y la parcialidad de la jornada laboral. Es, lo que Doringer y Piore (1983) definieron de forma brillante como la segmentación, bipolarización o dualización del mercado de trabajo. Desde este enfoque dual, el empleo estándar sería un sector primario de dicho mercado de trabajo caracterizado por la estabilidad, mejores condiciones laborales y mejores salarios, mientras que el atípico sería un mercado secundario de trabajo caracterizado por empleos mal pagados, inestabilidad laboral en alto grado $\mathrm{y}$, evidentemente, peores condiciones laborales.

Esa progresiva precariedad basada en la dualidad del mercado de trabajo se entiende mejor si tomamos en consideración las dimensiones que definirían dicha precariedad en relación a la percepción de los sujetos (trabajadores) que se ven afectada por ella. Así, hablaríamos de empleo precario cuando la posibilidad de perderlo es alta, cuando el control que tiene el trabajador para negociar con el empleador sus condiciones laborales es baja, cuando el nivel de protección del trabajador frente a despidos, cobertura y no discriminación laboral es inadecuado o, en el mejor de los casos, escaso y, finalmente, cuando el salario es bajo, el empleo se catalogaría como precario (Rodgers y Rodgers, 1992) y, más aún, si ese salario no evita el riesgo de pobreza y, con ello, una inserción laboral no segura.

\section{TRABaJAdor Y POBRE}

\section{El concepto de trabajador pobre}

La Organización Internacional del Trabajo (OIT) define el concepto de trabajador pobre dentro de un concepto más amplio como es el de subempleo. ${ }^{1}$ El subempleo puede ser considerado desde dos puntos de vista: $s u$ -

${ }^{1}$ En la Conferencia Internacional, celebrada en Ginebra en octubre de 1998, los Estadísticos del Trabajo aprueban una metodología para medir las situaciones de subempleo, así como las de empleo inadecuado. El subempleo se vincula a "insuficiencia de horas", siempre que las horas de trabajo de una persona ocupada sean insuficientes a las que desea desempeñar y está disponible para hacerlo. Junto a las situaciones de subempleo por insuficiencia de horas, se realizan otras recomendaciones de empleo inadecuado entendido éste como situaciones laborales que limitan las capacidades y el bienestar de los trabajadores. En este sentido, se alude al empleo inadecuado en relación a las competencias (subutilización del Capital Humano), el relacionado con los 
bempleo por insuficiencia de horas y por situaciones de empleo inadecuado (Mata, 1999). Con el primero de ellos, subempleo por insuficiencia de horas, nos referimos a aquellos que, en relación al periodo de referencia que se tome para medirlos, querrían trabajar más horas de las que lo hacen, estarían dispuestos a trabajar más horas de las que trabajan en ese periodo contemplado o, habrían trabajado menos de un cierto número de horas. Por el contrario, si hablamos de subempleo por situaciones de empleo inadecuado, estaríamos aludiendo a aquellas circunstancias en las que las personas estudiadas desearían cambiar su situación laboral actual o bien, están empleadas sí, pero ese empleo provoca situaciones que limitan sus capacidades y su propio bienestar. Es aquí precisamente donde encajaría el concepto de trabajador pobre desarrollado por la OIT, al señalar que entrarían en esa categoría aquellos trabajadores asalariados que tienen un nivel de renta que poco o nada se corresponde con las expectativas y características del trabajador. Hablamos de nivel de renta en términos de salario. Salarios que, por otra parte, son tan bajos que puede terminar provocando que ese trabajador en situación de subempleo trate de buscar empleos complementarios o abandonar el empleo que lo ha llevado a esa situación.

El Consejo Económico y Social Europeo (2009), en su Dictamen de 26 de febrero de 2009, define de forma muy clara, a partir de un indicador objetivo, qué podemos entender por trabajador pobre. Así, trabajador pobre será aquella persona que trabaja, al menos siete meses al año y que vive en un hogar cuya renta es inferior a 60 por ciento de la renta media nacional. Más allá del propio contenido del indicador, lo que vemos es que el trabajador pobre encaja en la idea de precariedad que hemos descrito más arriba, pero aporta una visión mucho más amplia, en la medida en que esa precariedad como consecuencia de la situación de subempleo alude a una doble dimensión. Por un lado, la individual (hablamos de una situación de pobreza activa) derivada de los recursos que provienen del trabajo y, por otro lado, el hogar, en la medida en que tomamos también como referencia al definir esta realidad los recursos provenientes de la familia o el propio nivel de vida del hogar del que forman parte este tipo de trabajadores.

Los niveles de desempleo se dispararon en España a partir de 2008, estando a día de hoy la tasa de paro aún por encima de 18 por ciento (según datos del primer trimestre del año 2017 aportados por la Encuesta de Población Activa (EPA). Esto altos niveles de desempleo son la nota característica del mercado de trabajo español, como es también la progre-

ingresos (ingresos limitados por baja productividad) y el relacionado con horarios de trabajo excesivos (personas empleadas que desean o buscan trabajar menos horas, ya sea en otro empleo o en el mismo empleo, aunque suponga una reducción de ingresos) (INE, 2017). 
siva precarización de las condiciones laborales. La Organización para la Cooperación y el Desarrollo Económico (OCDE) considera que para que podamos determinar el nivel de calidad o no de un empleo, tendremos que atender a aspectos como el nivel de salario, la seguridad laboral o la propia calidad del lugar de trabajo. Partiendo de este punto de vista, el trabajador pobre describe claras trayectorias laborales de precariedad puesto que vive en la inseguridad a razón de la inestabilidad que suele estar unida en su vínculo contractual, o por contar con jornadas laborales reducidas en comparación con lo que podría ser una jornada completa de trabajo y, todo ello, unido a la insuficiencia de los ingresos que vienen de las rentas de trabajo, así como otras condiciones laborales como pueden ser la equiparación con otra fuerza de trabajo (comparación con trabajadores más estables), movilidad funcional, movilidad geográfica, etc.

El trabajador pobre ve disminuida de forma considerable su capacidad para poder hacer frente a sus necesidades básicas, en la medida en la que los ingresos provenientes del trabajo son escasos o han ido disminuyendo progresivamente. La inestabilidad laboral, la parcialidad, la temporalidad, en definitiva, la falta de calidad en el trabajo incentivaría a este tipo de trabajador a buscar actividades informales (normalmente en la economía sumergida) o a solicitar ayudas públicas para poder dar respuesta a la insuficiencia de sus ingresos (Morata y Díaz, 2013).

El trabajador pobre vendría a ser caracterizado como aquel que presenta mayores niveles de desempleo y/o paro, periodos de permanencia en situaciones de desempleo muy largos y elevada rotación laboral en puestos de trabajo de similares características (Recio, 2007; García y Gutiérrez, 2011; Tejero, 2017).

\section{La negociación colectiva y el Estado de Bienestar}

La evolución y la incidencia de la desigualdad salarial a la hora de terminar produciendo esta categoría de trabajador pobre va a variar dependiendo de cuál sea el sistema de negociación colectiva del país, así como del modelo específico de Estado de Bienestar (Hans-Jürgen y Lohmann, 2008; García y Arranz, 2014).

Empezando por el sistema de negociación colectiva, este influirá tanto en la estructura de los salarios como en la propia dispersión de estos. Son dos las estrategias que incidirán en estos dos aspectos y en sentido positivo para el trabajador. Por una parte, mediante la implantación de tarifas de salario únicas para cada una de las categorías profesionales y, con ello, limitando la capacidad que puede tener el agente social empresa - la direc- 
ción empresarial para poder establecer diferencias salariales y, junto a ello, considerar como más importantes aspectos como es el caso de la experiencia o la propia antigüedad en el puesto de trabajo frente a la productividad individual. Y por otro lado, apostando por procesos de negociación colectiva que se funden en la coordinación de los diferentes agentes implicados en dichos procesos, coordinación que se completaría con la centralización de dicha negociación y, como consecuencia de ello, la aparición de una disminución de la desigualdad salarial entre empresas y sectores - ámbitos laborales (Machin y Van Reenen, 2007). Tal y como señala la OIT (2015) en aquellos países donde la negociación colectiva se ha centralizado y, por tanto, hay mayor número de trabajadores bajo el paraguas de convenios colectivos o de sector (y no tanto de empresa), la desigualdad salarial es menor o tiene menor incidencia.

En el caso español, al menos desde 2012, y como consecuencia de la Reforma Laboral de febrero de 2012 (Real Decreto 3/2012 de 10 de Febrero) el camino andado ha sido el contrario al que hemos descrito como más positivo en términos de desigualdad salarial, en un contexto de desregulación (Banyuls y Recio, 2017) de las relaciones laborales. Dicha reforma ha consagrado como alternativa en nuestro país la descentralización en el ámbito de la negociación colectiva. Esto es, se ha establecido la posibilidad de dar prioridad al convenio colectivo de empresa frente al convenio colectivo supra - empresarial. Los aspectos que se han dejado ahora en manos de dichos convenios de empresa son, por ejemplo, los salarios y su cuantía, las horas extraordinarias, el horario tanto laboral como la distribución de las vacaciones, el propio sistema de clasificación profesional así, y es uno de los aspectos más importantes, que la empresa pueda adaptar su política de contratación a sus propias necesidades (Morata y Díaz, 2013) de producción.

Junto con la negociación colectiva aludíamos a la influencia que puede tener el modelo de Estado de Bienestar en el propio nacimiento y afianzamiento de la categoría de trabajador pobre. Esping-Andersen (1990) parte de la idea de que la institución básica que configura la estratificación social en cualquier sociedad avanzada es el Estado de Bienestar. Diferencia entre tres regímenes de Estado de Bienestar. En primer lugar, estaría el Estado de Bienestar liberal. Este tipo de estado tiene un sistema de subsidios bajos asignados a aquellos que se comprueba que no tienen medios de supervivencia, algo que termina configurando una estructura social dual basada en la igualdad en la pobreza de una parte de la población (aquellos que viven de la protección social) y el bienestar para la mayoría de la población, ba- 
sado éste último en el mercado. Son ejemplos Estados Unidos, Canadá o Australia. El segundo modelo de Estado de Bienestar de Esping-Andersen es el de Estado de Bienestar Corporativista. Este modelo se basa en la conservación de las diferencias de estatus, función que ha terminado asumiendo el estado en una actitud corporativa frente al mercado, con lo que la función redistributiva se hace muy pequeña. Como ejemplos de este tipo de Estado de Bienestar tenemos a Austria, Francia, Alemania e Italia. Y, finalmente, el tercer modelo de Estado de Bienestar es el Estado de Bienestar Socialdemócrata. En este tipo de estados, los derechos sociales tienen un carácter universalista y no dependen del mercado. El Estado debe asegurar todos estos derechos, por lo que se convierte en el más igualitario de los tres modelos, siendo, por tanto, el que presenta mayor grado de movilidad social en la estructura social, en base a la garantía de la igualdad de oportunidades asegurada por el Estado. Entre los ejemplos más característicos de este tipo de Estado de Bienestar estarían Noruega y Suecia.

Pues bien, el modelo de Estado de Bienestar en España podríamos definirlo como mixto. Es decir, se trataría de un modelo a medio camino entre el socialdemócrata basado, como ya hemos indicado, en el universalismo y en la des-mercantilización de los derechos de tipo social y la extensión de los mismos al conjunto de los ciudadanos, y el modelo corporativista que, inspirado en el principio bismarckiano por el cual los trabajadores tendrán y recibirán una protección social desigual según sea la actividad laboral que desempeñen (y características de la misma como estabilidad y parcialidad), lo que terminará generando desigualdad dentro y entre el mismo colectivo general de trabajadores (Esping-Andersen, 1990). El modelo de Estado de Bienestar español ha sido clasificado también como modelo mediterráneo o familista, por cuanto la familia en nuestro país ha jugado, y lo sigue haciendo, un papel clave a la hora de proveer bienestar y como factor que ha permitido amortiguar las diferentes crisis económicas, entre ellas la que se ha vivido en España desde 2008.

\section{Las causas de la pobreza laboral}

Son diversas las causas que podrían explicar la existencia de pobreza laboral. Entre ellas señalaremos, a efectos de este trabajo, lo que conocemos como polarización del mercado de trabajo en cuando a un progresivo aumento de las desigualdades en términos salariales. Hablamos de una situación que se ha venido germinando en los mercados de trabajo a raíz de los cambios que se han producido como consecuencia del aumento de la demanda relativa de trabajadores cualificados o muy cualificados en los 
sectores relacionados con las Nuevas Tecnologías de la Información y la Comunicación (NTIC), contexto en el cual los trabajadores no cualificados de sociedades como la española han tenido más dificultades para competir con los trabajadores de las economías emergentes, estando en la base de todo ello los procesos de globalización económica así como los diferentes tratados internacionales (Atkinson, 2007; Machin y Van Reenen, 2007) para la liberalización del comercio, tratados que han permitido las deslocalizaciones y la irrupción de las transnacionales en el escenario de un nuevo mercado laboral global. Es decir, apuntamos al empobrecimiento de una masa laboral local como consecuencia negativa de la globalización.

Piketty (2014), por su parte, mantiene la idea de que cuando se produce un aumento de la desigualdad tanto en relación con los ingresos como a las rentas que provienen del trabajo. Ello es resultado de los cambios y transformaciones que se producen en la oferta y en la demanda tanto de habilidades como de cualificaciones en el contexto del mercado de trabajo. Junto a esto, indica que esa desigualdad de ingresos y rentas se explicaría también por la relación existente entre sistema educativo y tecnología o en relación con el propio acceso al sistema educativo.

La pobreza laboral, como ya hemos indicado al trabajar el concepto de trabajador pobre, toma dos dimensiones: la individual y la colectiva en términos de hogar. A nivel individual, el Informe Mundial de los Salarios (OIT, 2015) apuntaba en la dirección de una drástica caída de los salarios desde la crisis de 2008. Asimismo, en dicho informe, se indicaba que, aunque en 2010 pareció apreciarse una relativa recuperación, a continuación, y hasta 2013 (última fecha incluida en dicho informe) los salarios volvieron a experimentar una nueva caída. Es decir, estaríamos hablando de una pobreza laboral que no tendría la explicación tanto en la falta de crecimiento económico como en la existencia de desequilibrios estructurales (Banyuls y Recio, 2017)

Junto con estos ajustes salariales al albor del ciclo económico, hay otros aspectos que explicarían la presencia de pobreza salarial en esta dimensión individual. Este es el caso de la brecha salarial de género entendida ésta como la existencia de diferencias salariales por género como consecuencia de la presencia tanto de segregación horizontal (es decir, mayor presencia de mujeres en determinadas áreas de actividad tales como salud y servicios sociales) como segregación vertical (esto es, poca presencia de mujeres en cargos directivos de empresas) o la propia duración de la jornada laboral.

$\mathrm{Si}$ al ser mujer unimos situaciones en las que son las principales o únicas sustentadoras del hogar (llegamos así a la segunda dimensión), el ries- 
go de pobreza se hace mayor y, con ello, el riesgo de exclusión social como resultado de una combinación de brecha salarial y de falta de políticas sociales de apoyo a la infancia o en beneficio de la conciliación laboral y familiar. Se trataría de un riesgo de pobreza y de exclusión que no sólo quedaría marcado por la falta de ingresos o un bajo salario, sino por un más que probable alejamiento del mercado de trabajo y, con ello, la pérdida de parte de las funciones - características del trabajo que señalamos con anterioridad (Salanova et al., 1996), siendo la principal la pérdida de lazos sociales, una caída en sus posibilidades de participación social y, con ello, pérdida de una parte importante de sus propios derechos sociales (FOESSA, 2014).

García e Ibáñez (2007) profundizan en el impacto de los factores relacionados con el hogar e indican que es la estructura familiar la que parece contar con mayor capacidad explicativa y mayor influencia que las propias características que puedan tener los empleos (el factor individual). Hacen esta afirmación para señalar que no todos los trabajadores que tienen un salario bajo son pobres puesto que la inmensa mayoría vive en hogares en los que su salario no es el único, ni siquiera el más bajo, o viven en hogares en los que se percibe alguna ayuda pública o, simplemente, el papel de la familia en su función proteccionista es más que evidente (aludiendo al carácter familista del Estado de Bienestar, como señalamos con anterioridad). Son la intensidad laboral que tenga la unidad familiar junto con posibles cambios que se puedan producir en las rentas que provienen del trabajo, los factores que, en mayor medida, podrían estar incidiendo en la aparición y/o persistencia de situaciones de pobreza laboral.

García e Ibánez (2007) nos presentan una clasificación tremendamente útil a la hora de perfilar al trabajador pobre en relación con los propios factores de hogar señalando tres tipos de trabajador pobre. En primer lugar, aquel trabajador que vive en un hogar donde los ingresos son inferiores al umbral de pobreza y que reciben una remuneración baja por hora trabajada (entenderíamos remuneración baja en el caso de los asalariados aquel que cuenta con un salario /hora inferior a 60 por ciento de la mediana del salario / hora de la población asalariada). Un segundo tipo de trabajador pobre es aquel que teniendo un salario/hora dentro de la categoría bajo, cuenta con una estructura familiar que lo protege ante situaciones de riesgo. Serían, finalmente, también trabajadores pobres aquellos que no teniendo un salario/hora bajo, sí conviven en un hogar que calificaríamos como pobre como consecuencia de situaciones familiares de exclusión. 


\section{OBJETIVOS, HIPÓTESIS Y METODOLOGÍA}

El estudio que hemos desarrollado tiene por objetivo, más allá de la conceptualización y teorización desarrollada hasta el momento, perfilar algunas características que, en términos de las dos dimensiones individual y de hogar señaladas, caracterizarían al trabajador pobre en España. Partiendo de indicadores de condiciones de vida y de estructura salarial queremos validar la hipótesis de que, asumiendo que en nuestro país existe el trabajador pobre con anterioridad a la crisis que se inicia en 2008, el perfil de este se ha acentuado haciendo aún más profundas las distancias sociales entre los que ocupan esa posición en la estructura laboral y los que están lejos de ella.

Esta hipótesis se fundamenta en varios argumentos. Por una parte, consideramos que persisten las diferencias salariales atendiendo a variables como el sexo, la edad, el tamaño de hogar, la propia nacionalidad, el nivel de formación alcanzado. Por otra parte, han aumentado las distancias entre aquellos trabajadores que más ganan y los que perciben un salario menor. Decimos que persisten al menos en el periodo analizado que va desde 2008 hasta la fecha que las fuentes utilizadas así lo han permitido (2015).

El trabajo analítico que hemos desarrollado y que presentaremos a continuación, se ha realizado a partir de análisis estadístico descriptivo de datos de fuentes secundarias. En concreto hemos trabajado con la Encuesta de Condiciones de Vida con datos de las ediciones de 2008 a 2015 y con la Encuesta Anual de Estructura Salarial con datos desde 2008 a 2014. Establecemos el origen de la línea temporal en 2008 porque creemos que es el punto inicial en el que se comienzan a ver y registrar los efectos de la crisis económica y terminamos las series en el último dato publicado en el momento de la preparación de este artículo por el Instituto Nacional de Estadística (INE) en cada una de las encuestas mencionadas.

La Encuesta de Condiciones de Vida - ECV (INE, 2013) es un estudio que se encuadraría dentro de las operaciones armonizadas de tipo estadístico que, a nivel europeo, se desarrollan con el objetivo de contar con datos que permitan realizar labores de comparación interestatales en aspectos como la distribución de ingresos o la construcción de indicadores de exclusión social. Se trata de un estudio de tipo longitudinal y que permite seguir en el tiempo a un panel de personas fijo con la finalidad última de poder estudiar cómo se producen cambios en sus vidas y los efectos que tienen sobre ellas las modificaciones que se producen en las políticas de tipo socioeconómico. Se trata de una encuesta que, a efectos de nuestro estudio y 
objetivos, nos interesa porque no sólo se centra en la dimensión individual, sino que toma también el hogar como punto de referencia.

Por otra parte, hemos trabajado también con la Encuesta Anual de Estructura Salarial - EES (INE, 2012). Se trata de otra operación estadística que, con periodicidad anual, recoge información salarial, y donde la variable sexo se convierte en la central a analizar. Se trata de una encuesta cuyo antecedente más cercano es la Encuesta de Salarios en la Industria y los Servicios (también del INE). Se trata de una encuesta que complementa información proveniente de registros administrativos tanto de la Seguridad Social como del Impuesta de la Renta de las Personas Físicas, con una encuesta en la que se obtiene información directamente de las empresas. Para dar cuenta de la enorme cantidad de datos, indicaremos que, por ejemplo, en el año 2009 participaron en la encuesta un total de 26,956 empresas para un total de más de 230 mil trabajadores.

Antes de proceder a presentar resultados, señalaremos que hemos trabajado con la publicación y síntesis estadística que, de cada una de las fuentes, proporciona el INE. Las tablas se han generado inicialmente a través del Programa PC-AXIS aportado por el propio INE y, a continuación, han sido tratadas para su presentación en este artículo.

\section{Resultados}

\section{Renta media e indicadores de pobreza}

La crisis económica comienza a azotar con fuerza a la población española a partir del primer trimestre de 2008 (al menos en términos de destrucción de empleo). En toda lógica, deberíamos conocer en qué medida esos efectos de la crisis incidieron en la renta tanto de los ciudadanos españoles, como en la de la población extranjera residente en España.

Con datos de la ECV tomando como referencia el periodo completo y comparando el año 2008 con el 2015, la renta media de los nacionales españoles descendió 4.23 por ciento. Asimismo, no es hasta 2011 cuando comienza el verdadero descenso de este indicador puesto que, de los $11,779 €$ de renta media de 2008, se pasó a los 12,410 € en 2010 descendiendo a partir de entonces hasta los 11,281 € del año 2015.

El caso de la población extranjera es particularmente llamativo. En primer lugar, destacaríamos que los extranjeros, en todos los casos, cuentan con rentas medias por persona muy inferiores a las de los nacionales españoles. Mientras que entre estos últimos la renta media está siempre por encima de los 11 mil $€$, entre los extranjeros nunca ha superado los nueve 
mil $€$ (tomando el colectivo en conjunto: extranjeros de la Unión Europea - UE y extranjeros del resto del mundo). Por su parte, el comportamiento de la renta media anual por persona ha sido diferente dependiendo de si estamos ante población extranjera de la UE o ante población extranjera del resto del mundo. En el primero de los casos, entre la población extranjera de la UE con residencia en España, comenta que hay mucha variabilidad a lo largo del periodo analizado (unos años la renta es mayor, pasando a un año siguiente en el que baja, para volver a subir a continuación) si bien es el único grupo en el que se aprecia una subida de 2.1 por ciento en 2015 $(8,263 €)$ respecto a $2008(8,093)$. Sin embargo, el segmento peor parado en cuanto a renta media disponible por persona es el de los extranjeros del resto del mundo, quienes han pasado de casi siete mil $€$ en 2010 a poco más de 5,700 en 2015, dado este último que comparado con el primero de la serie $(6,268 €)$ supone una bajada de la renta media de casi nueve por ciento. Además, en 2015, la renta media por persona de los extranjeros del resto del mundo suponía 50 por ciento de la renta media anual por persona de un nacional español, cuando ese mismo porcentaje en 2008 era de 53.2 por ciento. Estamos pues, ante un escenario en el que el indicador de renta apunta hacia una desigualdad clara por nacionalidad, lo que unido a la caída de dicha renta (entre nacionales españoles y extranjeros del resto del mundo) señala una clara brecha de renta de tipo estructural en la sociedad española.

La estructura del hogar, como hemos indicado anteriormente, tiene su incidencia en la aparición de situaciones de riesgo que puedan llevar a un empobrecimiento (García y Sánchez, 2019) de la masa laboral. Son datos que nos pueden ir aproximando al conocimiento de este aspecto aquellos que se relacionan con la evolución de la renta media anual neta según el tamaño del hogar o número de miembros del mismo. Tomando como referencia de la ECV (Tabla 1), si comparamos la media global total comprobamos que se ha producido un importante empobrecimiento de la población de nuestro país en la medida en que ta dicha renta media neta se ha reducido de 2008 a 2015 en 9.36 por ciento pasando de $28,787 €$ a $26,092 €$ por hogar. Más allá de esta media global, lo especialmente llamativo es cómo ha evolucionado este aspecto según el número de miembros del hogar. Comprobamos que, entre 2008 y 2015, en los hogares de un solo miembro, no sólo no se ha producido una caída de la renta anual neta media, sino que se ha incrementado al final del periodo en casi un punto porcentual. 
Trabajadores pobles en España. El contexto de la crisis económica como marco para comprender ... / R. MARTÍNEZ et al.

Tabla 1: Renta anual neta media (en $€$ ) en España por tamaño del hogar (2008 y 2015)

\begin{tabular}{lrrrrrr}
\hline \multicolumn{7}{c}{ Número de miembros } \\
\hline Año & 1 & 2 & 3 & 4 & 5 o más & Total \\
\hline 2008 & 14,802 & 26,296 & 32,776 & 38,727 & 40,490 & 28,787 \\
2015 & 14,938 & 25,338 & 31,103 & 34,238 & 35,609 & 26,092 \\
$\%$ variación & 0.92 & -3.64 & -5.10 & -11.59 & -12.05 & -9.36 \\
$2008-2015$ & & & & & & \\
\hline
\end{tabular}

Fuente: elaboración propia a partir de la Encuesta de Condiciones de Vida - I.N.E.

Sin embargo, a partir de dos miembros en el hogar la caída de este indicador de renta es cada vez mayor, pasando de -3.64 por ciento de los hogares de dos miembros a -11.59 por ciento o -12.05 por ciento de los hogares de cuatro y cinco o más miembros respectivamente. El impacto del desempleo en España, con el aumento de la presencia de situaciones de paro entre miembros del hogar, ha tenido su impacto en la renta media, de tal forma que la intensidad laboral en los hogares se convierte en un factor explicativo de posiciones sociales tendentes a situaciones de pobreza, aumentando la probabilidad de pobreza en aquellos hogares donde exista una menor o más baja participación laboral de sus miembros (Tejero, 2017).

Uno de los indicadores más importantes, y que se calcula a partir de los datos de la ECV, es la tasa de riesgo de pobreza. La tasa de riesgo de pobreza en España viene a señalar el porcentaje de personas que viven en hogares cuya renta disponible equivalente es inferior al que se considera umbral de riesgo de pobreza para ese hogar. El propio INE (2013) define el umbral de pobreza a partir de las recomendaciones de Eurostat y lo sitúa en 60 por ciento de la mediana de los ingresos por unidad de consumo de las personas.

Pues bien, si analizamos el valor total (medio), la tasa de riesgo de pobreza en España ha aumentado desde 2008 a 2015 en 11.6 por ciento (Tabla 2), apareciendo notables diferencias atendiendo a la variable grupo de edad. Así, podemos comprobar cómo, mientras que en el grupo de menores de 16 años entre 2008 y 2015 esta tasa se ha incrementado en 7.5 por ciento, es entre los que tienen entre 16 y 29 años donde dicho riesgo se ha incrementado con más virulencia, pasando de 18.1 por ciento en 2008 a 29.2 por ciento en 2015 (lo que supone una variación de 61.3 por ciento). Esa misma tasa, y en el periodo estudiado, se ha incrementado en 23.9 por ciento entre los que tienen entre 30 y 44 años y en 40.8 por ciento entre los que tienen de 45 a 64 años. 
Tabla 2: Tasa de riesgo de pobreza en España por grupos de edad (2008 y 2015)

\begin{tabular}{lccr}
\hline Edad & 2008 & 2015 & $\begin{array}{r}\text { \% variación } \\
\text { 2008-2015 }\end{array}$ \\
\hline Menores de 16 años & 26.8 & 28.8 & 7.5 \\
Menos de 18 años & 27.3 & 29.6 & 8.4 \\
De 16 a 29 años & 18.1 & 29.2 & 61.3 \\
De 30 a 44 años & 17.6 & 21.8 & 23.9 \\
De 45 a 64 años & 15.2 & 21.4 & 40.8 \\
De 65 o más años & 25.5 & 12.3 & -51.8 \\
De 18 a 64 años & 16.5 & 22.8 & 38.2 \\
Total & 19.8 & 22.1 & 11.6 \\
\hline Fuente: elaboración propia a partir de la Encuesta de Condiciones de Vida - I.N.E.
\end{tabular}

De esta forma se confirmaría la idea de la influencia de la edad en la persistencia de la pobreza, siendo entre los más jóvenes dónde dicha persistencia podría ser mayor, en especial, en el caso de la persistencia de la pobreza laboral (Tejero, 2018). Así, tomando la edad como variable independiente, podemos relacionar la pobreza laboral con el desempleo juvenil (que alcanzó casi 50 por ciento de media nacional según la EPA en 2015) o con el desempleo en mayores de 45 años (en 2014, 44 por ciento del total de parados de España pertenecía al tramo de mayores de 45 años). En todo caso, resulta especialmente llamativo, que, entre los mayores, aquellos que tienen 65 o más años, no sólo no ha aumentado el riesgo de pobreza, sino que se ha visto reducido, pasando la tasa de 25.5 por ciento en 2008 a 12.3 por ciento en 2015. Sin duda, estamos ante el segmento de población que mejor ha mantenido su nivel adquisitivo en el periodo de crisis, puesto que son los menos afectados por el desempleo dada su situación mayoritaria de jubilación, lo que les ha asegurado unos ingresos estables (pensiones de jubilación) aún en la situación económica atravesada por nuestro país donde, como ya estamos viendo y podremos seguir comprobando más adelante, los salarios y las ganancias medias, en general, se han visto reducidas.

La tasa de riesgo de pobreza en España presenta también diferencias si atendemos al nivel formativo alcanzado por las personas (Tabla 3). Dentro de educación primaria o inferior (donde podríamos incluir una parte importante de esos mayores jubilados de los que acabamos de hablar) la tasa de pobreza entre 2008 y 2015 se ha reducido en 11.7 por ciento pasando de 28.1 a 24.8 por ciento, lo que viene a indicarnos lo importantes que están resultando las rentas por jubilación en nuestro país a la hora de amortiguar 
los efectos de la crisis. Sin embargo, las menores tasas de riesgo de pobreza estarían entre aquellos que tienen un nivel formativo superior. En este caso, salvo en 2014, dicha tasa no superó diez por ciento, habiendo pasado de 7.2 en 2008 a 9.8 por ciento en 2015 , lo que es un incremento de 36.1 por ciento, incremento similar al de los que tienen educación secundaria de segunda etapa, pero en este caso la tasa en 2008 era de 14.8 por ciento y en 2015 de 20.3 por ciento (más del doble que los de educación superior en ambos momentos señalados). Sin lugar a duda, son los que tienen educación secundaria de primera etapa los que peor situación han vivido, habiendo protagonizado un incremento de la tasa de riesgo de pobreza de más de 46 por ciento, situándose ésta en 2015 prácticamente en 30 por ciento. Así quedarían corroboradas algunas de las conclusiones de Tejero (2018) cuando indicaba que son aquellas personas con niveles de estudios no superiores los que presentarían un mayor riesgo de pobreza laboral temporal ${ }^{2}$ (con la excepción que se ha visto aquí de los que tienen el nivel de estudios más bajo y que se corresponderían con los más mayores en cuanto a edad).

Tabla 3: Tasa de riesgo de pobreza en España por nivel formativo alcanzado (2008 y 2015)

\begin{tabular}{lrrrrr}
\hline $\begin{array}{l}\text { Nivel } \\
\text { Formativo } \\
\text { Alcanzado }\end{array}$ & $\begin{array}{r}\text { Educación } \\
\text { primaria } 0 \\
\text { inferior }\end{array}$ & $\begin{array}{r}\text { Educación } \\
\text { secundaria } \\
\text { primera } \\
\text { etapa }\end{array}$ & $\begin{array}{r}\text { Educación } \\
\text { secundaria } \\
\text { segunda } \\
\text { etapa }\end{array}$ & $\begin{array}{r}\text { Educación } \\
\text { superior }\end{array}$ & Total \\
\hline 2008 & 28.1 & 20.4 & 14.8 & 7.2 & 18.6 \\
2015 & 24.8 & 29.9 & 20.3 & 9.8 & 21.0 \\
$\%$ variación & -11.7 & 46.6 & 37.2 & 36.1 & 12.9 \\
$2008-2015$ & & & & & \\
\hline
\end{tabular}

Fuente: elaboración propia a partir de la Encuesta de Condiciones de Vida - I.N.E.

En relación con la situación de actividad de la persona encuestada (esto es, si está ocupado, parado o jubilado) vemos como comienzan a confirmarse algunas de las afirmaciones que venimos realizando y aspectos que apuntan en la dirección de nuestras hipótesis (Tabla 4). Entre ocupados y parados se ha producido un aumento de la tasa de riesgo de pobreza, pasando ésta entre los primeros de 11.7 por ciento en 2008 a 14.8 por ciento de 2015 , mientras que, entre los parados, dicha tasa siendo de 31.3 por ciento en 2008 ha llegado a 44.8 por ciento en 2015 (lo que supone un incremento de más de 43 por ciento).

\footnotetext{
${ }^{2}$ Es pobreza temporal aquella que viven personas, familias y/o trabajadores, cuando "sufren etapa aisladas de pobreza" (Tejero, 2018: 2).
} 
Tabla 4: Tasa de riesgo pobreza en relación con la actividad (2008 y 2015)

\begin{tabular}{lrrrr}
\hline Año & Ocupados & Parados & Jubilados & Total \\
\hline 2008 & 11.7 & 31.3 & 20.4 & 18.6 \\
2015 & 14.8 & 44.8 & 10.3 & 21.0 \\
\% variación & 26.5 & 43.1 & -49.5 & 12.9 \\
$2008-2015$ & & &
\end{tabular}

Fuente: elaboración propia a partir de la Encuesta de Condiciones de Vida - I.N.E.

Es decir, tener empleo, estar ocupado en una tarea remunerada tanto por cuenta ajena como propia no elimina el riesgo de pobreza, pero, al menos, su crecimiento es menor respecto a los que viven situaciones de paro. Por otra parte, tenemos el caso de los jubilados cuyas tasas de riesgo de pobreza vienen a confirmar lo que hemos indicado a propósito de datos anteriores. En el caso de los jubilados, la tasa de riesgo de pobreza ha pasado de 20.4 por ciento en 2008 a 10.3 por ciento de 2015, lo que supone una reducción de estas de casi 50 por ciento, valor muy próximo al dato que presentábamos en relación con esta tasa para el grupo de edad de 65 o más. Hablaríamos también así de la persistencia del trabajador pobre (ocupado pobre) y como éste ha aumentado en el contexto de la crisis económica en España. De esta forma, la asociación clásica entre empleo - trabajo e inclusión social, el concebir el empleo como un mecanismo para la distribución de la riqueza y como una forma de conseguir ingresos suficientes para llevar una vida digna, se desdibuja de tal forma que el perfil de pobreza laboral ya no lo integran tanto los jubilados como los parados y ocupados (Banyuls y Recio, 2017).

Por umbral de riesgo de pobreza entendemos el nivel de ingresos a partir del cual una persona situada por debajo de ellos se encuentra en situación de riesgo de pobreza. En el contexto de la Unión Europea dicho umbral de riesgo de pobreza ha quedado señalado en 60 por ciento de la mediana de la renta disponible. A partir de la ECV podemos calcular ese umbral de riesgo de pobreza pero tomando en consideración el tipo de hogar (es decir, si hay o no niños en el hogar). En España, el umbral de riesgo de pobreza en un hogar de una única persona se situaría en torno a los ocho mil $€$ en el año 2015 , mientras que para un hogar de cuatro personas (dos de ellas adultos y las otras dos niños) dicho umbral no es de 32 mil $€$ (el resultante de cuatro veces el umbral de un hogar de una persona) sino algo más del doble de los ocho mil $€$ de un hogar unipersonal. Esteban y Losa (2015) explican muy bien esta aparente contradicción. Señalan que, para satisfacer las necesida- 
des de un hogar de cuatro personas no es necesaria una renta que sea cuatro veces mayor que la de un hogar de una única persona, estando la explicación en las economías de escala asociadas a la convivencia y en el menor coste que tiene la satisfacción de las necesidades de los niños de tal forma que "para satisfacer las necesidades del segundo y restantes miembros adultos, sólo se requiere la mitad de la renta que si ese adulto viviese solo (...)" mientras que para la "satisfacción de las necesidades de los restantes miembros menores de 14 años solo se precisa, aproximadamente, la tercera parte de la renta del primer adulto" (Esteban y Losa, 2015: 10). Además, y tal y como indica Tejero (2018), es en aquellos hogares en los que hay una mayor existencia de menores que son dependientes donde la probabilidad de vivir situaciones de pobreza recurrente (aquella que se da cuando se sale y se entra en la pobreza varias veces) y pobreza persistente (aquella que se da cuando se está en situación de pobreza durante varios años) es mayor.

Un aspecto que forma parte de nuestras hipótesis es que han aumentado las distancias en términos de renta entre la población española y, puesto que la mayor parte de la renta viene por la vía del trabajo si han aumentado esas distancias es porque algún segmento de población se ha empobrecido más o, por el contrario, algún otro se ha enriquecido más. Una forma de aproximarnos a la validación de esta hipótesis es la que nos permite el cálculo de la ratio S80/S20 que no es más que el cálculo del cociente de los ingresos totales de 20 por ciento de las personas que cuentan con ingresos mayores entre los ingresos totales de 20 por ciento de personas que cuenta con ingresos menores. Es también conocido como el ratio quintil (quintil superior de distribución de la renta entre el quintil inferior y que para España se puede calcular con la ECV). En el caso de España, en el periodo contemplado, se ha producido un aumento del cociente quintil superior entre quintil inferior. Así, mientras que en 2008 la renta de 20 por ciento de población con ingresos más altos multiplicaba por 5.6 la renta de 20 por ciento de la población con menor renta, en 2015, esa ratio era ya de 6,9, lo que supone un incremento de más de 23 por ciento. Esa diferencia en la distribución de la renta es muy superior a lo que sucede en el conjunto de la Unión Europea de los 15 donde, y con datos de Eurostat, si bien también ha habido incremento, ese ha sido menor que en España. Por ejemplo, dicha ratio era en 2014 de 5.2 en la UE-15, mientras que en España era ya de 6.8. Por tanto, en el caso de España, hablamos de un claro aumento de la desigualdad de renta entre aquellos que más ingresos tienen respecto a los que menos. 
Finalmente, un concepto interesante y que nos permite ahondar en la idea de la pobreza laboral es el de hogar con baja intensidad de trabajo. Un hogar de baja intensidad de trabajo es aquel en el que los miembros que están en edad de trabajar (el INE lo calcula sólo para hogares con personas de 0 a 59 años) trabajaron menos de 20 por ciento de su potencial total de trabajo durante el año anterior al momento en el que se realiza la encuesta, momento que se toma como referencia para los ingresos (INE, 2013). En definitiva, de lo que estamos hablando es de hogares en los que se da situación de subempleo. Para este estudio hemos podido conseguir datos de porcentaje de personas viviendo en hogares con baja intensidad de trabajo tanto por grupos de edad (Tabla 5) como por sexo (Tabla 6).

Tabla 5: Porcentaje de personas viviendo en hogares con baja intensidad de trabajo por grupos de edad (2008 y 2015)

\begin{tabular}{lrrr} 
Edad & 2008 & 2015 & \% variación 2008-2015 \\
\hline Menores de 16 años & 4.0 & 11.6 & 190.0 \\
Menos de 18 años & 4.2 & 12.0 & 185.7 \\
De 16 a 29 años & 6.1 & 18.4 & 201.6 \\
De 30 a 44 años & 4.7 & 11.9 & 153.2 \\
De 45 a 64 años & 11.7 & 20.4 & 74.4 \\
De 18 a 64 años & 7.3 & 16.5 & 126.0 \\
Total & 6.6 & 15.4 & 133.3 \\
\hline
\end{tabular}

Fuente: elaboración propia a partir de la Encuesta de Condiciones de Vida - I.N.E.

Tabla 6: Porcentaje de personas viviendo en hogares con baja intensidad de trabajo por sexo (2008 y 2015)

\begin{tabular}{lrrr}
\hline Año & Hombres & Mujeres & Total \\
\hline 2008 & 6.1 & 7.0 & 6.6 \\
2015 & 15.8 & 15.1 & 15.4 \\
\% variación & & 115.7 & 133.3 \\
$2008-2015$ & 159.0 & & \\
\hline
\end{tabular}

Fuente: elaboración propia a partir de la Encuesta de Condiciones de Vida - I.N.E.

Comenzado por el porcentaje de personas que viven en hogares con baja intensidad laboral por grupos de edad (Tabla 5) indicaremos que se ha producido un más que notable incremento entre 2008 y 2015 en esta proporción en todos los grupos de edad. El incremento ha sido superior a 
150 por ciento en todos los grupos de edad hasta los 44 años, pasando, por poner un ejemplo, de un porcentaje de cuatro por ciento de personas menores de 16 años que vivían en hogares con baja intensidad de trabajo en 2008 a un porcentaje de 11.6 por ciento en 2015 (incremento de 190 por ciento). En el caso del grupo de 16 a 29 años, el porcentaje ha pasado de 6.1 por ciento en 2008 a 18.4 por ciento en 2015 (incremento de 201.6 por ciento), mientras que entre los que tienen de 30 a 44 años en 2008 el porcentaje de personas que vivía en este tipo de hogares era de 4.7 por ciento pasando a 11.9 por ciento en 2015 (incremento de 153.2 por ciento).

Y según el sexo la persona encuestada (Tabla 6), comprobamos como también se han producido variaciones diferenciadas. Si bien los hombres partían de una situación algo mejor que las mujeres (en 2008 el porcentaje de hombres que vivía en hogares con baja intensidad de trabajo era de 6.1 frente a siete por ciento de las mujeres) al final del periodo analizado, el porcentaje de hombres que vivía en este tipo de hogares es mayor que en el porcentaje de mujeres (15.8 por ciento de ellos, frente a 15.1 por ciento de ellas), lo que supondría un incremento de 159 por ciento en el caso de los hombres frente a un incremento de 115.7 por ciento en el de las mujeres.

En definitiva, como señala Tejero (2018), son aquellos factores explicativos importantes desde un punto de vista estático, como es el caso de factores del hogar (tipo, tamaño) y la existencia o no de participación laboral de los miembros de ese hogar, los que nos permiten tener una perspectiva más clara en el momento del análisis de la existencia o no de una pobreza crónica. Hablar de baja intensidad laboral en el hogar, para el caso español, supone comprender cómo se relacionan los elementos de tipo laboral con el riesgo de pobreza de aquellos que forman parte de la población ocupada, y como dicho riesgo ha aumentado como consecuencia de la crisis económica desde 2008 (Lope, 2015).

\section{Los salarios}

Hasta ahora nos hemos centrado, sobre todo, en la dimensión del hogar $\mathrm{y}$ en aspectos que pueden incidir en la aparición de riesgos asociados a la pobreza laboral. En su momento señalamos que el propio concepto de trabajador pobre incluía una dimensión más de tipo individual. Para validar las hipótesis de esa dimensión individual es por lo que nos vamos a detener, continuación, en indicadores centrados en el salario y en la ganancia en términos de recursos provenientes del trabajo.

En España se utiliza, como en otros países del contexto de la OCDE un salario de referencia: el Salario Mínimo Interprofesional (SMI) que lo que 
viene a señalar es el mínimo que debe recibir un trabajador como retribución a su trabajo en el marco de una jornada legal y sin atender a distinción de sexo, edad, tipo de jornada o tipo de contrato. En nuestro país, es el Gobierno de España quien fija cada año cuál va a ser el valor del SMI y se publica por vía de Real Decreto en el Boletín Oficial de Estado. Para establecer dicho importe lo que se toma como referencia es el índice de precios al consumo (IPC), la propia productividad media nacional que se haya conseguido alcanzar, así como el posible incremento que exista de la participación del trabajo en el conjunto de la renta nacional.

Pues bien, tomando el SMI como dato, hay que indicar que este ha crecido en 60 por ciento en los últimos 15 años. Así, de los $442.2 €$ de 2002 la cuantía del SMI está fijada el año 2017 en 707.6. En todo caso, en 2008, dicho SMI era de $600 €$ pasando a los $648.6 €$ en 2015 (lo que supone un incremento de 8.1 por ciento en el periodo más complejo de la crisis económica).

El SMI es una referencia que podemos tomar como marco a la hora de establecer diferencias por sexo y, con ello, si hay más o menos probabilidad de existencia de pobreza laboral asociada al sexo según el porcentaje de trabajadores en función de su ganancia con respecto al SMI (Tabla 7). Podemos comprobar que no hay grandes cambios en los porcentajes entre el año 2008 y el año 2014, pero lo que sí resulta llamativo es la distribución por sexo y cómo se van invirtiendo los porcentajes a medida que aumenta el número de SMI que se toman como ganancia. Por ejemplo, de 100 por ciento de personas cuyo salario va de 0 a 1 SMI en 2008 (esto es, de 0 a 600 $€)$, casi 70 por ciento son mujeres mientras que 30 por ciento son hombres. Sin embargo, si hablamos de ganancias superiores a ocho SMI, la situación se invierte de tal manera que, de 100 de los que ganaban más de 4,800€ al mes 75.9 por ciento eran hombres y frente a 24.1 por ciento que eran mujeres. Es evidente la enorme brecha salarial que se puede entrever con estos datos. Es más, si nos detenemos una a una en las cifras presentadas, en ningún momento se aproximan los porcentajes de hombres y mujeres, salvo en 2008 y para entre uno y dos SMI, igualdad que se rompe en 2014. En este último año analizado, son más mujeres que hombres (en términos de porcentaje) en situaciones de dos veces el SMI o inferior, pero son siempre más los hombres en situaciones de dos o más veces el SMI. De esta forma, comprobamos cómo el porcentaje de salarios bajos es mucho más alto entre las mujeres que entre los hombres, no habiéndose producido grandes cambios a este respecto en el periodo analizado, lo que nos lleva a pensar hasta qué punto esta brecha salarial es estructural al mercado de 
trabajo español en términos de feminización de esos salarios bajos (Banyuls y Recio, 2017).

Tabla 7: Porcentaje de trabajadores en función de su ganancia con respecto al Salario Mínimo Interprofesional (SMI) por sexo (2008 y 2014)

\begin{tabular}{lrr}
\hline 2008 & Mujeres & Hombres \\
\hline De 0 a 1 SMI & 69.85 & 30.15 \\
De 1 a 2 SMI & 50.92 & 49.08 \\
De 2 a 3 SMI & 37.47 & 62.53 \\
De 3 a 4 SMI & 36.10 & 63.90 \\
De 4 a 5 SMI & 34.35 & 65.65 \\
De 5 a 6 SMI & 31.45 & 68.55 \\
De 6 a 7 SMI & 26.24 & 73.76 \\
De 7 a 8 SMI & 28.04 & 71.96 \\
Más de 8 SMI & 22.70 & 77.30 \\
\hline 2014 & Mujeres & Hombres \\
\hline De 0 a 1 SMI & 68.61 & 31.39 \\
De 1 a 2 SMI & 54.33 & 45.67 \\
De 2 a 3 SMI & 42.23 & 57.77 \\
De 3 a 4 SMI & 39.63 & 60.37 \\
De 4 a 5 SMI & 36.50 & 63.50 \\
De 5 a 6 SMI & 33.97 & 66.03 \\
De 6 a 7 SMI & 32.39 & 67.61 \\
De 7 a 8 SMI & 30.75 & 69.25 \\
Más de 8 SMI & 24.11 & 75.89 \\
\hline Fuente: elaboración propia a partir de la Encuesta Anual de Estructura Salarial - I.N.E.
\end{tabular}

Hay muchas formas de generar información que nos alumbre en la dirección deseada al propósito de este artículo. Una de ellas es contemplar la distribución de los datos atendiendo a diferentes medidas de tendencia central y/o posición. Nos estamos refiriendo a la media, la mediana, los percentiles y cuartiles. Para el análisis, comparación y establecimiento de diferencias entre años y en relación con variables como el sexo, este tipo de medidas son sumamente interesantes. Así, en la Tabla 8, presentamos la ganancia media anual por trabajador (expresada en euros), pero también la expresamos según percentil 10 y 90, cuartil inferior ( 25 inferior) y cuartil 
superior ( 25 superior) así como mediana (que nos permitiría estimar el salario bajo propio del trabajador pobre como 60 por ciento de la mediana del salario medio). Todos estos datos se presentan por sexo y para el periodo 2008 a 2014.

Tabla 8: Ganancia anual por trabajador (en $€$ ) en media, percentiles, cuartiles y medianas (2008 y 2014)

\begin{tabular}{lrrrrrr}
\hline Sexo & Media & $\begin{array}{r}\text { Percentil } \\
10\end{array}$ & $\begin{array}{r}\text { Cuartil } \\
\text { inferior }\end{array}$ & Mediana & $\begin{array}{r}\text { Cuartil } \\
\text { superior }\end{array}$ & $\begin{array}{r}\text { Percentil } \\
90\end{array}$ \\
\hline 2008 & & & & & & \\
Mujeres & $18,910.62$ & $7,088.36$ & $11,038.28$ & $15,886.02$ & $23,446.18$ & $34,397.13$ \\
Hombres & $24,203.33$ & $11,594.27$ & $15,160.00$ & $20,057.56$ & $29,385.82$ & $41,792.77$ \\
Total & $21,883.42$ & $8,903.52$ & $13,342.53$ & $18,244.40$ & $26,874.02$ & $38,558, .9$ \\
2014 & & & & & & \\
Mujeres & $19,744.82$ & $6,205.32$ & $10,900.70$ & $16,771.32$ & $25,134.57$ & $36,627.29$ \\
Hombres & $25,727.24$ & $10,242.05$ & $15,688.20$ & $21,528.50$ & $31,837.00$ & $45,259.58$ \\
Total & $22,858.17$ & $7,626.20$ & $13,217.84$ & $19,263.78$ & $28,782.70$ & $41,350.36$ \\
\% variación $2008-2014$ & & & & & \\
Mujeres & 4.41 & -12.46 & -1.25 & 5.57 & 7.20 & 6.48 \\
Hombres & 6.30 & -11.66 & 3.48 & 7.33 & 8.34 & 8.30 \\
Total & 4.45 & -14.35 & -0.93 & 5.59 & 7.10 & 7.24 \\
\hline Fuente: elaboración propia a partir de la Encuesta Anual de Estructura Salarial - IN.E. &
\end{tabular}

En primer lugar vemos que la media de ganancia anual entre 2008 y 2014 y por trabajador ha pasado de $21,883 €$ a $22,858 €$ lo que supondría un incremento de 4.5 por ciento. Sin embargo, podemos ver con más claridad la falta de representatividad de los datos que, a veces, arroja una media cuando no es capaz de capar la dispersión de los mismos. Si nos damos cuenta, mientras que la media parece apuntar a una mejoría de la situación en cuanto a ganancia media por trabajador, al detenernos en los percentiles y en los cuartiles comprobamos cómo en el percentil diez de la distribución de trabajadores que menos ganan no se ha producido dicho incremento de la ganancia media, sino que lo que se ha producido ha sido una reducción de la ganancia anual media de los trabajadores de ese percentil de 14.4 por ciento pasando de 8,903€ en 2008 a 7,626 € en 2014. Por el contrario, los trabajadores del percentil 90 son los que de verdad han influido en esa apa- 
rente subida media del conjunto de los trabajadores españoles por cuanto entre ellos, la ganancia media anual en 2008 era de 38,558 € pasando en 2014 a 41,350 € (lo que es un incremento de 7.24 por ciento). Esto nos indica que la mejoría en la situación económica que comienza a apreciarse justo en el final de la serie analizada no afectaba por igual a todos los trabajadores. Mientras que los que estaban en peor situación al inicio de la crisis, no han mejorado, los que estaban en mejor posición salarial, sí han visto cómo su situación ha mejorado. En este caso, lo que se aprecia es que los ingresos han bajado, pero no para todos y, como vimos con anterioridad, las situaciones de pobreza han aumentado. A este respecto, Laparra y Pérez (2012) aluden a cómo la crisis económica que se inicia en 2008 ha tenido una especial incidencia en los que denominan colectivos más vulnerables, razón por la cual, muchas personas y familias cada vez han encontrado mayores dificultades para poder satisfacer sus necesidades.

Tomado en consideración la variable sexo ahondamos aún más en las desigualdades (Tabla 8). Las mujeres siempre cuentan con una ganancia media inferior al hombre en todas y cada una de las medidas calculadas (media general, percentiles y cuartiles) así como en cuanto a la mediana, siendo mayores a su vez las caídas que se producen en las ganancias medias y, por el contrario, menores los incrementos (entre 2008 y 2014) que se presentan en los cuartiles y percentiles superiores. La mediana, como hemos indicado, es un buen referente para aproximarnos a la idea de salario bajo. Si calculamos 60 por ciento de la mediana del salario en nuestra distribución de datos podemos decir que, para el conjunto de la población en 2008, sería un salario bajo un importe medio de 10,946€, mientras que una mujer con salario bajo en 2008 cobraría de media 9,531 $€$ y un hombre 12,034 € de media. Ese mismo cálculo para 2014 quedaría así: salario bajo medio global por importe de $11,558 €$, salario bajo medio para las mujeres por importe de 10,062 € y para los hombres por importe de $12,917 €$. En ambos casos, como podemos comprobar, ese salario bajo se situaría por debajo del cuartil inferior (25 por ciento de los que menos ganan) pero estaría por encima del percentil diez (diez por ciento de los que menos ganan). Así, nos ponemos en otra dirección como consecuencia del salario diferenciado: la calidad percibida del empleo. El salario diferenciado, por ejemplo por sexo, como hemos visto, tendría una influencia en la percepción que los trabajadores tendrían de la calidad de su trabajo, en la medida en que el ingreso, la retribución, es una dimensión presente en cualquier intento de medir dicha calidad percibida (Díaz, Ficapal y Torrent, 2015). 
La dualización del mercado de trabajo (Doeringer y Piore, 1983), la segmentación del mismo se materializa, entre otros aspectos, en la seguridad en el puesto de trabajo. Dicha seguridad se define fundamentalmente por la duración del empleo. En la Tabla 9 presentamos la ganancia media anual por trabajador (en euros) según si el contrato es indefinido o es temporal (duración determinada) y lo hacemos tanto a nivel global como por sexo.

Tabla 9: Ganancia media anual por trabajador $(e n €)$ según tipo de contrato y sexo (2008 y 2014)

\begin{tabular}{lrrr}
\hline Tipo de contrato & Mujeres & Hombres & Ambos sexos \\
\hline 2008 & & & \\
Duración indefinida & $20,008.48$ & $26,329.00$ & $23,544.24$ \\
Duración determinada & $15,069.12$ & $17,062.62$ & $16,204.29$ \\
Total & $18,910,62$ & $24,203.33$ & $21,883.42$ \\
2014 & & & \\
Duración indefinida & $21,147.36$ & $27,999.69$ & $24,746.47$ \\
Duración determinada & $14,661.47$ & $16,691.14$ & $15,680.55$ \\
Total & $19,744.82$ & $25,727.24$ & $22,858.17$ \\
\% variación 2008-2014 & & & \\
Duración indefinida & 5.69 & 6.35 & 5.11 \\
Duración determinada & -2.71 & -2.18 & -3.23 \\
Total & 4.41 & 6.30 & 4.45 \\
\hline
\end{tabular}

Fuente: elaboración propia a partir de la Encuesta Anual de Estructura Salarial - I.N.E.

Lo primero que nos podría sorprender es que la comparación 2008 2014 (en la ganancia media por sexo) parece haberse producido un incremento tanto para el caso de los hombres como para el caso de las mujeres siendo este algo mayor para los hombres (6.3 por ciento de incremento entre los hombres frente a 4.41 por ciento entre las mujeres). Sin embargo, este dato ocultaría una realidad muy interesante al objeto de nuestro estudio: ese incremento se ha dado porque donde ha subido en términos medios la ganancia media anual ha sido tanto entre los trabajadores como entre las trabajadoras con contrato de duración indefinida, mientras que, entre los que tenían un contrato de duración determinada (es decir, temporal) lo que se ha producido es una reducción de la ganancia media de -2.2 por ciento en el caso de los hombres y de 2.7 por ciento en el de las mujeres. 
Así, es evidente, que el tipo de contrato ahonda en las desigualdades producidas por el mercado de trabajo en nuestro país, de tal forma que la dualización de dicho mercado laboral, con un aumento de la precariedad laboral y la presencia constante de la flexibilización (García y Martínez, 2012) se hace cada vez más profunda (al menos en el contexto temporal estudiado). Banyuls y Recio (2017) relacionarían la temporalidad del contrato y la duración de este con la existencia de salarios bajos e ingresos de tipo global bajo como seña de identidad del mercado de trabajo español, contexto en el que podrían darse situaciones en las que, teniendo incluso una jornada laboral normal (completa), los ingresos podrían llegar a ser insuficientes cuando la duración de los contratos es mayoritariamente temporal (de duración determinada). Por su parte, Tejero (2018) señala que, para España, el tipo de contrato tiene influencia en el tipo de pobreza que se puede experimentar, de tal forma que los trabajadores con contrato indefinido tienen menos probabilidades de caer en riesgo de pobreza laboral temporal, mientras que los trabajadores a tiempo parcial tienen más riesgo de pobreza recurrente.

Finalmente, cabe preguntarnos por cuál es verdaderamente la proporción de trabajadores con ganancia baja (en términos del INE) o salario bajo (tal y como lo venimos manejando en este trabajo). En la Tabla 10 podemos ver cómo dicha proporción se ha mantenido en torno a 17-19 por ciento entre los años 2008 y 2013, con la excepción de 2010 que es de 13.4 por ciento y habiendo bajado a 12.9 por ciento en el año 2014. Junto con ese importante dato (en el año 2012 era trabajador pobre casi dos de cada diez trabajadores españoles) tenemos la proporción de mujeres en el total de asalariados con ganancia baja en cada uno de los años analizados. El dato es especialmente sorprendente y valida la hipótesis de feminización de la pobreza laboral (Tejero, 2018): en todos los años analizados (de 2008 a 2014) sobre el total de trabajadores con salario bajo las mujeres siempre han representado entre 64 y 66 por ciento. 
Tabla 10: Proporción de trabajadores (en \%) con ganancia baja y proporción de mujeres (en \%) sobre el total de asalariados con ganancia baja (2008-2014)

\begin{tabular}{lrr}
\hline Año & $\begin{array}{r}\text { Trabajadores con } \\
\text { ganancia baja }\end{array}$ & $\begin{array}{r}\text { Mujeres en el total de asalariados } \\
\text { con ganancia baja }\end{array}$ \\
\hline 2008 & 17.6 & 64.0 \\
2009 & 18.0 & 64.3 \\
2010 & 13.4 & 66.0 \\
2011 & 18.9 & 64.1 \\
2012 & 17.2 & 64.9 \\
2013 & 17.9 & 64.4 \\
2014 & 12.9 & 63.8 \\
$\%$ variación 2008-2014 & -26.6 & -0.3 \\
\hline Fuente: elaboración propia a partir de la Encuesta Anual de Estructura Salarial - I.N.E.
\end{tabular}

\section{Conclusiones}

En este trabajo hemos reflexionado, tomando como marco analítico, la triple perspectiva señalada al inicio. Así, hemos podido conocer en qué medida la biografía personal de los individuos, las desigualdades inherentes al propio mercado de trabajo español y las diferencias en la posición social de dichos individuos y de los hogares en la estructura social, podría estar en el origen de la categoría trabajador pobre en el contexto de nuestro país.

Creemos que los datos seleccionados para enfocar el estudio han sido los correctos puesto que nos han permitido delimitar escenarios de riesgo social, riesgo que, llevado al extremo, pondría en peligro la propia estabilidad del sistema social. Esto es así, en la medida que el trabajador pobre enfrenta la realidad con mayores dificultades que la otra parte de los trabajadores que, de forma más o menos segura, han podido completar su proceso de inserción laboral y, con ello, su proceso de inserción social. Es decir, existe un riesgo claro de desaparición de la que fue siempre (en la sociedad industrial) la relación positiva entre inclusión social y trabajo (Lope, 2015)

El trabajo presentado ha cumplido con el objetivo de perfilar la situación de subempleo que supone el estado de precariedad laboral del trabajador pobre. De esta forma, hemos trabajado desde el punto de vista de la pobreza activa, desde los recursos que vienen del trabajo y en términos individuales, así como en las situaciones sobrevenidas a raíz de la estructura de los hogares españoles y su umbral de pobreza. 


\section{Trabajadores pobles en España. El contexto de la crisis económica como marco para comprender ... / R. MARTÍNEZ et al.}

En primer lugar, hemos podido comprobar que la nacionalidad actúa como un factor de desigualdad social. La renta media anual por persona es muy inferior en el caso de los extranjeros que viven en España y no son de la UE, frente a la renta de los nacionalizados españoles. Diferencia de renta que puede llegar a ser de más de 50 por ciento. La nacionalidad actuaría, así como factor a contemplar a la hora de definir el perfil de trabajador pobre.

La tasa de riesgo de pobreza, indicador claro de situaciones de precariedad laboral, ha empeorado y mucho a lo largo del periodo de crisis económica analizado, especialmente entre la población activa y los menores de 16 años. Sólo entre los mayores de 65, jubilados en su mayoría, la tasa de riesgo de pobreza ha mejorado de 2008 a 2015. Desempleo juvenil y situaciones de paro de larga duración en mayores de 45 años, son realidades que incidirían de forma muy negativa en las condiciones de vida de jóvenes y adultos por encima de los 40 años. Pero no sólo la edad ha aparecido como factor diferenciador en términos de tasa de riesgo de pobreza, también lo ha sido el nivel formativo alcanzado (tasas de riesgo de pobreza menores entre los que tienen nivel formativo - educativo superior) o por situación de actividad (aumento de las tasas de riesgo de pobreza tanto entre ocupados y parados, pero con mayor incidencia en los segundos). Así, las variables sociodemográficas como edad y nivel de estudios explicarían datos diferenciados en términos de pobreza laboral (Tejero, 2018).

Entre 2008 y 2015 se ha producido en España un importante aumento de las distancias entre aquellos ciudadanos de rentas más altas y los de rentas más bajas. Así, tal y como hemos comentado a lo largo de nuestros resultados, en 2015 la renta de 20 por ciento de población española con más ingresos era casi siete veces mayor que la de 20 por ciento de menores ingresos, distancia que ha ido aumentando en el periodo analizado. De igual forma, y en este sentido de aumento de las desigualdades, el porcentaje de personas que viven en hogares con baja intensidad de trabajo se ha incrementado notablemente en todos los grupos de edad y en relación al sexo, lo que indica que han aumentado de forma destacada las situaciones de subempleo en nuestro país. La estructura del hogar en España va a tener, como hemos visto, una incidencia clara en la probabilidad de existencia de situaciones tendentes o que puedan desembocar en el empobrecimiento (García y Sánchez, 2019).

Los salarios y su estudio en relación a la influencia que puedan tener en la aparición de trabajadores pobres también han formado parte de nuestro trabajo. Un primer punto de referencia es el Salario Mínimo Interprofe- 
sional y, en concreto, las enormes diferencias descubiertas en por sexo en relación con el mismo tomado como ganancia del trabajador. Así, casi siete de cada diez trabajadores que ganan menos de un SMI son mujeres, mientras que tres cuartas partes de los trabajadores que tienen como ganancia más de ocho SMI son hombres. Enormes diferencias y distancias salariales que dejan clara la persistente brecha salarial por género que sigue caracterizando a nuestro país.

Si bien la ganancia media por trabajador ha mejorado en el periodo 2008 - 2014, dicha media oculta las verdaderas desigualdades de la distribución de salarios. Si vamos a los percentiles, hemos comprobado como la ganancia media anual de diez por ciento de personas que menos ganan en España se ha visto reducida en dicho periodo en 14.4 por ciento, frente al percentil 90, donde está diez por ciento de los que tienen mayor ganancia, que ha visto incrementada su ganancia media anual en 7.24 por ciento. Los datos analizados indican también que las diferencias se mantienen cuando la variable sexo entra en juego, contando las mujeres siempre con una ganancia menor sea cual sea el indicador que se calcule (media, mediana, percentil o cuartil). Así mismo, hemos podido comprobar, que las diferencias salariales se mantendrían también por situaciones de precariedad marcadas por contratos de duración determinada (contratos temporales) frente a los contratos indefinidos, habiéndose reducido más la ganancia media entre 2008 y 2014 en las trabajadoras mujeres con contrato temporal que entre los trabajadores hombres.

En definitiva, hemos podido comprobar la presencia casi coyuntural y sistémica de la pobreza laboral en nuestro país y, junto a ello, la feminización de dicho proceso de empobrecimiento de la masa laboral. En plena crisis económica, casi 20 por ciento de los trabajadores españoles tenía un salario bajo (era trabajador pobre) y de ellos, en torno al 64-65 por ciento eran mujeres.

Creemos que hemos sabido describir una realidad poco halagüeña de nuestro mercado de trabajo. Hemos comprobado como la atipicidad de los empleos, la precariedad como signo distintivo, cada día pone en más riesgo la inserción laboral de una parte importante de los trabajadores de nuestro país, pudiendo así ver como hay una relación significativa entre desempleo y la existencia o no de más o menos probabilidad de caer en lo que Andrade y Jiménez (2018) denominan la trampa de la pobreza. Además, si el salario, la ganancia, la renta media nos acerca al umbral de pobreza poco o nada podremos hacer en términos de las funciones que cumple el trabajo. En definitiva, este artículo puede ser una muestra del enorme camino que 
nos queda a todos para revertir situaciones nada deseables en sociedades teóricamente avanzadas, pero puede servir de ayuda, como estudio de la pobreza que es, como fuente para comprender las desigualdades de tipo social (Zugasti y Laparra, 2017).

\section{REFERENCIAS BIBLIOGRÁFICAS}

Andrade, Luis Antonio y Jiménez, Carlos Alberto, 2018, "El desempleo y la probabilidad de caer en trampas de pobreza: consideraciones para países en vías de desarrollo", en Revista Española de Investigaciones Sociológicas, núm, 164, pp. $3-20$.

Atkinson, Anthony Barnes, 2007, "Disparidad salarial en los países de la OCDE", en Revista Internacional del Trabajo, vol. 126, núm. 1-2, pp. 45-68.

Banyuls, Josep y Recio, Albert, 2017, "Pobreza laboral en España: causas y alternativas políticas", en Anuario IET de Trabajo y Relaciones Laborales, vol. 4, pp. 135-149.

Bericat, Eduardo, 1989, "Cultura productiva y desarrollo endógeno. El caso andaluz", en Revista de Estudios Regionales, núm. 24, pp. 15-43.

Consejo Económico y Social Europeo, 2009, "Dictamen del Comité Económico y Social Europeo", del 26/02/2009, sobre el tema "Trabajo y pobreza: hacia un enfoque global indispensable", en Diario Oficial de la Unión Europea, C 318, del 26/02/2009, pp. 1-5.

Dahrendorf, Ralf, 2003, "El nuevo subproletariado", en Díaz Salazar, Rafael (ed.), Trabajadores precarios. El Proletariado del S.XXI. Ediciones HOAC, Madrid, pp. 127-135.

De Miguel, Jesús M., 1998, Estructura y cambio social en España, Alianza Editorial, Madrid

Díaz, Ángel, Ficapal, Pilar y Torrent, Joan, 2015, "Determinantes multidimensionales en la calidad percibida del empleo. Evidencia empírica para España", en Revista Internacional de Sociología, núm. 73 (1), pp. 2-14.

Doeringer, Peter B. y Piore, Michael J., 1983, "El paro y el mercado dual de trabajo", en Toharia, Luis (comp). El mercado de trabajo: Teorías y aplicaciones. Lecturas seleccionadas, Alianza Editorial, Madrid, pp. 307-320.

Esping-Andersen, Gøsta, 1990, Three Worlds of Welfare Capitalism. Polity Press, Cambridge.

Esteban, Miguel A. y Losa, Antonio, 2015, Guía básica para interpretar los indicadores de desigualdad, pobreza y exclusión social, EAPN España, Madrid.

FOESSA, 2014, VII Informe sobre exclusión y Desarrollo Social en España, Fundación Fomento de Estudios Sociales y de Sociología Aplicada (FOESSA), Madrid. 
García, Carlos y Arranz, José M., 2014, "Evolución de la desigualdad salarial en los países desarrollados y en España en los últimos treinta años", en FOESSA, VII informe sobre exclusión y desarrollo social en España. FOESSA, Madrid.

García, Beatriz y Sánchez, María Carmen, 2019, "Medición de la pobreza subjetiva en España y su localización espacial", en Revista Española de Investigaciones Sociológicas, núm. 165, pp. 83-100.

García, Isabel y Gutiérrez, Rodolfo, 2011, "Persisting inequalities in a growing employement context", en Fraser, Neil; Gutiérrez, Rodolfo y Peña, Ramón (eds). Working poverty in Europe: a comparative approach, Palgrave - McMillan, Hampshire, pp. 133-154.

García, Isabel e Ibáñez, Marta, 2007, "Los trabajadores pobres y los bajos salarios en España: un análisis de los factores familiares y laborales asociados a las distintas situaciones de pobreza", en Empiria. Revista de metodología de ciencias sociales, núm.14, pp. 41-67.

García, José Manuel y Martínez, Rafael, 2012, "Ser joven hoy en España. Dificultades para el acceso al mundo de los adultos", en Barataria - Revista Castellano - Manchega de Ciencias Sociales, núm. 14, pp. 29-40,

Giner, Salvador, 1979, Sociología. Ediciones Península, Barcelona.

Hans-Jürgen, Andreß. y Lohmann, Henning, 2008, The Working Poor in Europe. Chentelhan, Edward Elgar.

INE, 2012, Encuesta anual de estructura salarial (EAES). Metodología. INE, Madrid.

INE, 2013, Encuesta de Condiciones de Vida (ECV). Metodología. INE, Madrid.

INE, 2017, Encuesta de Población Activa (EPA). Metodología. INE, Madrid.

Laparra, Miguel y Pérez, Begoña, 2012, Crisis y fractura social en Europa. Causas y efectos en España. Colección de Estudios Sociales, 35. Obra Social La Caixa, Barcelona.

Lope, Andreu, 2015, "El trabajo y la inclusión social: una relación en peligro", en Revista Lavboratorio, núm. 26, pp. 108-129

Machin, Stephen y Van Reenen, John, 2007, Changes in Wage Inequality. Centre for Economic Performance, London School of Economics and Political Science, 18.

Mata, Adriana, 1999, Definiciones internacionales y futuro de las estadísticas del subempleo, Oficina Internacional del Trabajo, Ginebra.

Morata, Belén y Díaz, María Teresa, 2013, "Reforma laboral en España: precariedad, desigualdad social y funcionamiento del mercado de trabajo", en Estudios Socio-Jurídicos, núm.15, pp. 41-71.

OIT, 2015, Informe Mundial sobre Salarios 2014/2015. Salarios y desigualdad de ingresos. Organización Internacional del Trabajo, Ginebra. 
Piketty, Thomas, 2014, Capital in the twenty-first century. Harvard University Press, Harvard.

Recio, Albert, 2007, “¿Trabajar evita la pobreza? Bajos salarios en el mercado laboral español”, en Documentación social, núm. 143, pp. 31-44.

Requena, Félix, 2001, Amigos y redes sociales: elementos para una sociología de la amistad, CIS - Siglo XXI, Madrid.

Rodgers, Gerry y Rodgers, Janine, 1992, El trabajo precario en la regulación del mercado laboral. Crecimiento atípico en la Europa Occidental. Ministerio de Trabajo y Seguridad Social, Madrid.

Salanova, Marisa; Hontangas, Pedro y Peiró, José M., 1996, “Motivación Laboral", en Peiró, José. M y Prieto, Fernando (eds). Tratado de Psicología del Trabajo, vol. 1. Editorial Síntesis, Madrid. pp. 215-249.

Sarriés, Luis, 1999, Sociología Industrial. Las relaciones industriales en la sociedad postmoderna. Mira Editores, Zaragoza.

Tejero, Aroa, 2017, "Permanencia en la pobreza laboral: la influencia de la pobreza pasada en la Presente", en Revista Española de Investigaciones Sociológicas, núm. 157, pp. 141-162.

Tejero, Aroa, 2018, "Pobreza laboral en España. Un análisis dinámico”, en Revista Internacional de Sociología, núm. 76(2), pp. 2-18

Valenzuela, Hugo; Reygadas, Luis y Cruces, Francisco, 2015, "Mi trabajo es mi vida. La incrustación de los mundos de la vida y del trabajo en las empresas españolas", en Revista Española de Investigaciones Sociológicas, núm. 150, pp. 191210

Zugasti, Nerea y Laparra, Miguel, 2017, "Midiendo la pobreza a nivel autonómico en España. Una propuesta reflexiva" en Revista Española de Investigaciones Sociológicas, núm. 158, pp. 117-136.

\section{RESUMEN CURRICULAR DE LOS AUTORES}

\section{Rafael Martínez Martín}

Licenciado en CC.PP. y Sociología y Doctor en Ciencias Políticas y Sociología por la Universidad de Granada. Ha sido Vicedecano de Grado, Postgrado y Prácticas en la Facultad de Ciencias Políticas y Sociología de la Universidad de Granada (2008-2012); profesor invitado en L' École des Hautes Études en Sciences Sociales de París . Actualmente es Profesor Titular del Departamento de Sociología en Universidad de Granada. Entre sus últimas publicaciones se encuentran: Training for employment: an analysis of its busines sector context in spain (2015), El desempleo y la precariedad laboral como conflicto social: análisis del contexto laboral 
español (2016), La orientación laboral como política activa de empleo en Andalucía (2017), Sociología Laboral. Los cambios en el mundo del trabajo y los nuevos retos laborales del S.XXI (2018).

ORCID: https://orcid.org/0000-0003-3941-5011

Dirección electrónica: rmmartin@ugr.es

José Manuel García Moreno

Licenciado en Ciencias Políticas y Sociología y Doctor en Sociología por la Universidad de Granada. Máster en Márketing y Gestión Comercial por ESIC. En la actualidad es Profesor del del Departamento de Sociología de la Universidad de Granada. Primer Premio Nacional de Terminación de Estudios en Sociología 2001 por el Ministerio de Educación. Entre sus publicaciones destacan: Transnational Firms as an Example of Industrial Relocation: Tijuana (Mexico) and Tangier - Tetouan (Morocco) (En coautoría con A. Trinidad y M. Solís), Economía, Trabajo y Empleo (En coautoría con R. Martínez) y ¿Conflicto de identidad? Una discusión sobre las dimensiones identitarias de la juventud en España (en coautoría con L. Feliciano).

ORCID: https://orcid.org/0000-0003-2429-8764

Dirección electrónica: josemgar@ugr.es

Antonio M. Lozano Martín

Licenciado en Filosofía y Ciencias de la Educación y Doctor en Sociología por la Universidad de Granada; profesor invitado en el Centre de Recherche sur les Liens Sociaux. Université Paris 5-René Descartes-, 2013; profesor invitado en el área de Scienze dell'Investigazione de la Universitá degli Studi dell'Aquila (Italia), 2016. Actualmente es Profesor del Departamento de Sociología de la Universidad de Granada y Vicedecano de Ordenación Académica y RRII de la Facultad de Trabajo Social. Entre sus publicaciones destacan "Tratado de mediación en solución de conflictos" (en coautoría con G. Orozco, J.L. Monereo y R. González); "Estudio sobre los procesos Étnicos, Culturales y Sociolingüisticos y su relación con los conflictos multiétnicos en Kazajistán" (en coautoría con Botagoz Rakiecheva) y "Sociología del conflicto en las sociedades contemporáneas" (en coautoría con Diego Becerril).

ORCID: https://orcid.org/0000-0002-8250-4266

Dirección electrónica: lozanoma@ugr.es

Artículo recibido el 24 de mayo de 2017 y aprobado el 26 de octubre de 2018. 